\title{
NONFACTORIZATION IN SUBSETS OF THE MEASURE ALGEBRA
}

\author{
J. T. BURNHAM
}

Abstract. In this note we unify and simplify some recent results showing the impossibility of factoring in certain convolution subalgebras of the group algebra of a nondiscrete LCAG. A new result is a direct proof of nonfactorization of the classical Hardy spaces, regarded as convolution algebras, on the circle. By considering the ideal of Hilbert-Schmidt operators in the algebra of compact operators on a Hilbert space we illustrate that nonfactorization is not peculiar to convolution.

It is known that the group algebra of a LCAG has the factorization property. The general case is due to Cohen [5], while Rudin [13], [14], solved the problem for locally Euclidean $G$. In 1939, Salem [15] obtained the same result for the circle group.

Recently, [10], [18], [17], ${ }^{1}$ attention has been given to the impossibility of factoring by convolution in subalgebras of group algebras. Most of these nonfactorization results are for explicit choices of Reiter's Segal algebras [12]. For further details on history and related results see [8], [9].

It may be of interest to keep in mind recent generalizations of Segal algebras due to Burnham [2], [3] and Cigler [4].

Our objective is to present simple proofs of some of the known nonfactorization results by exploiting a single idea (Lemma $\mathrm{A}$ ). We implicitly use the fact that if $G$ is a nondiscrete LCAG [fixed for the remainder of this note], then as a consequence of Theorem 1 in [6], $\exists f \in C_{c}(G)$ with $\hat{f} \notin L^{1}(\hat{G})$.

Several of the known nonfactorization results are a simple consequence of

Lemma A. Let $(X, \mathscr{F}, \mu)$ be a positive measure space. Let $f$ be a bounded complex-valued function on $X$ with $f \in L^{p}(\mu)$ for some $p \in(1, \infty)$. Iffor each

Received by the editors August 27, 1971 and, in revised form, January 12, 1972.

AMS 1969 subject classifications. Primary 4255; Secondary 4265.

Key' words and phrases. Convolution, measure algebras, factorization, Banach algebras, Segal algebras, normed ideals.

${ }^{1}$ This was brought to our attention after an earlier draft of this paper had been accepted for publication. There is some overlap in material and the results in [17] are more comprehensive but the techniques of the present note are sufficiently different from those in [17] to warrant this note. In particular our result concerning the Hardy spaces is not included in [17].

C American Mathematical Society 1972 
positive integer $n$, we can write $f=f_{1} \cdots f_{n}$ (pointwise multiplication) with $f_{i}$ bounded and $f_{i} \in L^{p}(\mu)$, then $f \in L^{1}(\mu)$.

Proof. Since $f$ is bounded, $f \in L^{t}$ for $t \geqq p$. Choose $t=n \geqq p$ and $a_{i}=1 / n$. Since $\left|f_{i}\right|^{t} \in L^{1}$ we have by the generalized Hölder inequality,

$$
\int_{X}\left(\left|f_{1}\right|^{t}\right)^{a_{1}} \cdots\left(\left|f_{n}\right|^{t}\right)^{a_{n}} d \mu \leqq\left.\left\|\left|f_{1}\right|^{t^{\prime}\left\|_{1}^{a_{1}} \cdots\right\|}\right\| f_{n}\right|^{t} \|_{1}^{a_{n}}<\infty .
$$

But the left side of the inequality is precisely $\|f\|_{1}$ so the proof is complete. Here are our nonfactorization results (we write $A^{2}$ for $A * A$ ).

Theorem B. If $\hat{A} \not L^{1}(\hat{G})$ but $\hat{A} \subset L^{p}(\hat{G})$ for some $p \in(1, \infty)$, then $A^{2} \neq A$.

Proof. Suppose $A^{2}=A$. Then for each $g \in A$ and positive integer $n$ we can write $g=g_{1} * \cdots * g_{n}$. Taking Fourier-Stieltjes transforms and applying Lemma A we obtain the contradictory fact $\hat{g} \in L^{1}(\hat{G})$.

Corollary C. If $p \in(1, \infty)$, then $A_{p}(G)=\left\{f \in L^{1}(G) \mid \hat{f} \in L^{p}(\hat{G})\right\}$ fails to factor. More generally, if $\eta$ is a positive measure on $\hat{G}$, then $A_{p}(G, \eta)$ fails to factor.

THEOREM D. Let $\hat{A} \notin L^{1}(\hat{G})$. If $A \subset L^{p}(G)$ for some $p \in(1,2]$ or if $A \subset L^{1}(G) \cap L^{p}(G)$ for some $p>1$, then $A^{2} \neq A$.

This is also proved by applying Lemma A, using the Hausdorff-Young theorem and Plancherel's theorem.

Corollary E. $L^{1}(G) \cap L^{p}(G)$ fails to factor for all $p \neq 1$.

Corollary F. Identify $G$ with $[0,2 \pi)$. If $p \in(1, \infty)$, then $H^{p}(G)$ fails to factor.

Proof. By a result of Boas [1], $L^{p}$ and $H^{p}$ are topologically isomorphic ( $H^{p}$ is given the inherited $L^{p}$ norm). Corollary E applied to the explicit construction in [1] gives the result.

Example. Let $C=$ ideal of compact operators on some Hilbert space. Let $B=$ ideal of Hilbert-Schmidt operators (in $C$ ) with the HilbertSchmidt norm. Then [3] $B$ is an $A$-Segal algebra in $C$. Moreover $B^{2}=$ trace class $\subsetneq B$. It would be interesting to know if the (proper) $A$-Segal algebras of [2] fail to factor. An affirmative answer would settle, in particular, the problem for all (proper) Segal algebras: a plausible conjecture is that all proper symmetric Segal algebras [11, p. 17] fail to factor.

Remark. In [7], [16], it is shown that $M_{0}(G)$ fails to factor by delicate ideal theoretic arguments. It would be of interest to have a direct proof of this fact within the context of the techniques of this note. 
Finally, I would like to thank the referee for correcting many egregious errors in the first draft of this paper. His comments have been most helpful, and $I$ am indeed grateful.

\section{REFERENCES}

1. R. P. Boas, Isomorphism between $H^{p}$ and $L^{p}$, Amer. J. Math. 77 (1955), 655-656. MR 17, 1080.

2. J. T. Burnham, Closed ideals in subalgebras of Banach algebras. I, Proc. Amer. Math. Soc. 32 (1972), 551-555.

3. - Closed ideals in subalgebras of Banach algebras. II: Ditkin's condition (in preparation); Notices Amer. Math. Soc. 17 (1970), 815. Abstract \#70T-B149.

4. J. Cigler, Normed ideals in $L^{1}(G)$, Nederl. Akad. Wetensch. Proc. Ser. A $72=$ Indag. Math. 31 (1969), 273-282. MR 40 \#3327.

5. P. Cohen, Factorization in group algebras, Duke Math. J. 26 (1959), 199-205. MR 21 \#3729.

6. R. E. Edwards, On functions which are Fourier transforms, Proc. Amer. Math. Soc. 5 (1954), 71-78. MR 15, 633.

7. C. C. Graham, $M_{0}(G)$ is not a prime ideal of measures, Proc. Amer. Math. Soc. 27 (1971), 551-562.

8. E. Hewitt, The ranges of certain convolution operators, Math. Scand. 15 (1964), 147-155. MR 32 \#4471.

9. E. Hewitt and K. A. Ross, Abstract harmonic analysis. Vol. II: Structure and analysis for compact groups analysis on locally compact Abelian groups, Die Grundlehren der math. Wissenschaften, Band 152, Springer-Verlag, New York and Berlin, 1970. MR 41 \#7378.

10. J. C. Martin and L. Y. H. Yap, The algebra of functions with Fourier transforms in $L^{p}$, Proc. Amer. Math. Soc. 24 (1970), 217-219. MR 40 \#646.

11. H. Reiter, $L^{1}$-algebras and Segal algebras, Lecture Notes in Math., no. 231, Springer-Verlag, Berlin and New York, 1971.

12. - Classical harmonic analysis and locally compact groups, Oxford Math. Monographs, Clarendon Press, Oxford, 1968.

13. W. Rudin, Factorization in the group algebra of the real line, Proc. Nat. Acad. Sci. U.S.A. 43 (1957), 339--340. MR 19, 46.

14. - Representation of functions by convolutions, J. Math. Mech. 7 (1958), 103115. MR 21 \#3728.

15. R. Salem, Sur les transformations des séries de Fourier, Fund. Math. 33 (1939), $108-114$.

16. N. Th. Varopoulos, Sur les mesures de Radon, d'un groupe localement compact abélien, C. R. Acad. Sci. Paris 258 (1964), 3805-3808. MR 28 \#4386.

17. H.-C. Wang, Non-factorization in group algebras, Studia Math. (to appear); See also, Ph.D. Dissertation, University of Iowa, Iowa City, Iowa, 1971.

18. L. Yap, Ideals in subalgebras of the group algebras, Studia Math. 35 (1970), 165175. MR 42 \#4968.

Department of Mathematics, University of Iowa, Iowa City, Iowa 52240 